\title{
Fenômenos psíquicos e o problema mente-corpo: notas históricas sobre uma tradição conceitual negligenciada
}

\section{Psychic phenomena and the mind-body problem: historical notes on a neglected conceptual tradition}

\author{
Carlos S. Alvarado ${ }^{1}$ \\ 1 Visiting Scholar, Rhine Research Center, Durham, North Carolina, Estados Unidos.
}

Este artigo é uma versão revista e reduzida do capítulo de C. S. Alvarado, "Psychic phenomena and the mind-body problem: historical notes on a neglected conceptual tradition", publicado em: Moreira-Almeida A, Santos FS. Exploring frontiers in the mind brain relationship. Springer: New York; 2012. p. 35-51.

Tradução: Ronald Kleinsorge Roland

Revisão Técnica: Alexander Moreira-Almeida

Recebido: 15/6/2013 - Aceito: 17/6/2013

\begin{abstract}
Resumo
Embora exista uma longa tradição de discussões filosóficas e históricas sobre o problema mente-corpo, a maioria delas não menciona os fenômenos psíquicos como tendo implicações para esse tópico. Este artigo é uma revisão de escritos selecionados publicados na literatura dos séculos XIX e XX sobre o mesmerismo, o espiritualismo e a pesquisa psíquica cujos os autores discutiram sobre aparições, telepatia, clarividência, experiências fora do corpo e outros fenômenos parapsicológicos como evidência para a existência de um princípio separado do corpo e responsável pela consciência. Os autores discutidos são de diferentes períodos de tempo. Entre eles estão John Beloff, J. C. Colquhoun, Camille Flammarion, J. H. Jung-Stilling, Frederic W. H. Myers e J. B. Rhine. Mais do que defender a validade de sua posição, minha proposta é documentar a existência de uma tradição intelectual e conceitual que tem sido negligenciada por filósofos, entre outros, nas discussões sobre o problema mente-corpo e aspectos de sua história.
\end{abstract}

Alvarado CS / Rev Psiq Clín. 2013;40(4):157-61

Palavras-chave: Relações mente-corpo, parapsicologia, percepção extrassensorial, aparições, mediunidade.

\begin{abstract}
Although there is a long tradition of philosophical and historical discussions of the mind-body problem most of them make no mention of psychic phenomena as having implications for such an issue. This article is an overview of selected writings published in the XIX and XX centuries literatures of mesmerism, spiritualism, and psychical research whose authors have discussed apparitions, telepathy, clairvoyance, out-of-body experiences and other parapsychological phenomena as evidence for the existence of a principle separate from the body and responsible for consciousness. Some writers discussed here include individuals from different time periods. Among them are John Beloff, J. C. Colquhoun, Camille Flammarion, J. H. Jung-Stilling, Frederic W. H. Myers, and J. B. Rhine. Rather than defend the validity of their position, my purpose is to document the existence of an intellectual and conceptual tradition that has been neglected by philosophers and others in their discussions of the mind-body problem and aspects of its history.
\end{abstract}

Alvarado CS / Rev Psiq Clín. 2013;40(4):157-61

Keywords: Mind-body relations, parapsychology, extrasensory perception, apparitions, mediumship.

\section{Introdução}

Existe uma história longa de especulações e escritos sobre a mente e suas várias relações postuladas com o corpo ${ }^{1-4}$. Ela envolve, entre outras, ideias descrevendo e conceituando a mente como um epifenômeno ou como um princípio independente das funções corporais.

Neste artigo eu não tentarei identificar as várias soluções propostas para compreensão da relação mente-corpo ao longo do tempo. Em vez disso, minha proposição é focar em uma tradição intelectual, geralmente negligenciada, cujos representantes defenderam a existência de um princípio independente do corpo baseado na existência do então chamado fenômeno psíquico, supranormal ou parapsicológico tais como as aparições, a mediunidade, a clarividência e a telepatia. Além disso, meu foco será nos escritos dos séculos XIX e XX.

\section{Fenômenos psíquicos}

Tais fenômenos adentraram a era moderna ocidental por meio de uma variedade de movimentos, entre eles o mesmerismo e o espiritualismo ${ }^{5,6}$, para não mencionar muitas outras crenças e práticas vindas do passado ${ }^{7,8}$. Muitos livros tratam de fenômenos psíquicos que desafiam as visões materialistas nas quais a mente (ou espírito ou alma, dependendo da abordagem de cada autor) poderia transcender o corpo físico e assim mostrar sua independência do corpo. São exemplos Crowe's The night-side of nature ${ }^{9}$, Kardec's Le livre des esprits $^{10}$ e Perty's Die mystischen Erscheinungen der menschlichen Natur ${ }^{11}$. Esses foram esforços iniciais que suplementaram as especulações filosóficas dualistas existentes sobre a mente ou o espírito.

Embora ocorrências referidas como fenômenos psíquicos venham sendo registradas desde a Antiguidade12,13, estudos mais sistemáticos têm sido desenvolvidos em épocas mais recentemente. Além do mesmerismo (séculos XVIII e XIX) e do espiritualismo (séculos XIX e XX), a pesquisa psíquica se desenvolveu no último quarto do século XIX. Em 1882 a Sociedade de Pesquisa Psíquica (em inglês: Society for Psychical Research - SPR) foi fundada em Londres com a proposta de apresentar "uma tentativa organizada e sistemática de investigar o grande grupo de fenômenos controversos designados por termos como mesmérico, psíquico e espiritualista" 14 . Enquanto a SPR tinha diversos espiritualistas como membros que 
contribuíram para o desenvolvimento da Sociedade, o que tornou essa organização diferente foi que ela também tinha muitos membros acadêmicos proeminentes. Isto incluía acadêmicos da Universidade de Cambridge, tais como o filósofo Henry Sidgwick (1838-1900) e o classicista e poeta Frederic W. H. Myers (1843-1901). Além desses, entre outros indivíduos eminentes associados com a SPR, estavam os físicos William Barrett (1844-1925) e Balfour Stewart (1828-1887) e o político Arthur Balfour (1848-1930), que mais tarde se tornou primeiro ministro. Em anos posteriores muitos homens eminentes se tornaram presidentes da SPR, incluindo como exemplos o psicólogo e filósofo americano William James (1842-1910), o químico e físico inglês William Crookes (1832-1919), o físico inglês Oliver Lodge (1851-1940), o fisiologista francês Charles Richet (1850-1935) e o filósofo francês Henri Bergson (1859-1941). Paralelamente ao estudo da telepatia espontânea, membros da SPR estudaram telepatia por meio de experimentos e analisaram casos de casas assombradas, médiuns e aparições de indivíduos falecidos ${ }^{15}$. A SPR também patrocinou estudos e discussões sobre fenômenos dissociativos e a mente subconsciente ${ }^{16}$. Mas havia também estudos vindos de outros países, entre eles a França e a Alemanha ${ }^{17,18}$.

Alguns dos indivíduos envolvidos nesses estudos, apoiavam a noção de que a mente era um princípio separado do corpo. Um dos líderes da SPR, o acima mencionado acadêmico clássico Frederic W. H. Myers, viu seu trabalho a partir dessa perspectiva. De fato, ele afirmava que: "Nós defendemos firmemente... que a atitude, os hábitos mentais, os métodos através dos quais a ciência física tem crescido de modo profundo e amplo, deveriam ser também aplicados ao mundo espiritual 19 " (p. 117).

Essa questão foi abordada no trabalho de William James, que considerou a pesquisa psíquica uma abordagem empírica válida para o estudo da mente. Como visto no trabalho de James ${ }^{20,21}$ com a médium Leonora E. Piper (1857-1950), e em outros trabalhos, James acreditava que os estudos empíricos dos fenômenos psíquicos eram importantes para o entendimento da consciência. Isso o levou a se tornar envolvido com o trabalho na SPR, da qual foi presidente em 1896, e com a fundação e as primeiras pesquisas da Sociedade Americana de Pesquisa Psíquica (American Society for Psychical Research) ${ }^{22,23}$.

No seu livro Human Immortality, James ${ }^{24}$ discutiu as ideias de "transmissão e produção" como explicações para a consciência. Tais conceitos se referiam, respectivamente, à independência da mente em relação ao corpo e ao epifenomenalismo. O primeiro era a suposição de que a mente se manifestava por meio do sistema nervoso, mas era um princípio independente, enquanto no segundo a ideia era de que a mente era produzida pelo sistema nervoso. Na sua visão os fenômenos psíquicos eram consistentes com a teoria da transmissão.

Mas, independentemente de tais ideias, gostaria de focar dois aspectos nesta seção. Primeiro, o ceticismo prevalente no passado sobre essas ideias e em segundo a tendência recente nos escritos históricos sobre tópicos psicológicos.

Enquanto alguns indivíduos acreditavam na existência dos fenômenos psíquicos e usavam tais manifestações para promover a crença em uma natureza espiritual da humanidade, tais interpretações não eram compartilhadas por muitos, e certamente não pela maioria da comunidade científica. Os leitores desse artigo deveriam estar cientes de que esse tópico era tão controverso no passado quanto é no presente ${ }^{25}$. Muitos dos autores do século XIX explicavam os relatos desses fenômenos usando explicações tão convencionais como fraude, coincidências, alucinações, ilusões, sugestão e uma variedade de processos psicofisiológicos relacionados à histeria e à hipnose ${ }^{16,29}$. Havia muitas tentativas de reduzir o fenômeno em termos de patologia, um tópico explorado por alguns em relação à mediunidade ${ }^{30-33}$.

Independentemente de tal ceticismo geral, leitores interessados deveriam compreender que o tópico que discutimos segue as tendências vistas na historiografia da psicologia e da psiquiatria. Começando com Ellenberger ${ }^{34}$, tem havido vários estudos que têm feito uma boa defesa da ideia de que os fenômenos psíquicos e o seu estudo têm sido um fator significativo no desenvolvimento de ideias sobre a mente. Conceitos tais como dissociação e mente subconsciente foram apoiados no passado por meio da referência a fenômenos como transes e automatismos dos médiums ${ }^{29-35}$ (veja também várias revisões ${ }^{30-36}$ ). A pesquisa psíquica representou mais do que interesse no supranormal. Muitos dos seus pesquisadores também tocaram na questão da natureza da mente, suas camadas e suas separações ${ }^{16,38}$. Os autores desse recente trabalho mudaram visões previamente existentes sobre o fenômeno psíquico e sobre movimentos tais como a pesquisa psíquica. Em vez de verem esses tópicos como meras superstições, ou como obstáculos no desenvolvimento das ideias sobre a mente, essa nova erudição acadêmica coloca a pesquisa psíquica e similares como catalizadores e importantes fatores contribuidores para os estudos empíricos que significativamente afetaram os campos da psicologia e da psiquiatria.

No restante deste artigo farei um sumário do uso dos fenômenos psíquicos feito pelas literaturas do mesmerismo, do espiritualismo e da parapsicologia na defesa da ideia de uma mente independente. Meu ponto não é defender a existência separada da mente em relação ao corpo. Em vez disso, pretendo mostrar a existência de uma literatura negligenciada nas discussões históricas do problema mente-corpo, ligando fenômenos psíquicos à ideia de que a mente (e o espírito) é separada do corpo. Essa literatura é variada em termos de argumentos apresentados. Algumas ideias são derivadas de trabalhos empíricos concretos, enquanto outras são menos empiricamente embasadas. Mas em todos os casos elas são baseadas em observações de fenômenos cuja interpretação levou os autores que citarei a acreditarem que o pressuposto materialista é uma explicação insuficiente.

\section{Mesmerismo e a mente não física}

O mesmerismo foi um dos primeiros movimentos em larga escala a trazer a atenção do mundo acadêmico ocidental para os fenômenos psíquicos. Começando no século XVIII e, posteriormente, no XIX, o mesmerismo produziu muita literatura relacionada aos fenômenos psíquicos $5,36,38,39$.

O nome desse movimento tem origem em Franz Anton Mesmer (1734-1815) ${ }^{40}$, o qual catalisou essas ideias no século XVIII. O mesmerismo foi um movimento baseado na ideia de uma força universal chamada magnetismo animal, conectada ao corpo humano e responsável por muitas das manifestações, entre elas o sonambulismo magnético (transe) e as curas. Embora existissem muitas conceituações diferentes dessa força - um princípio geralmente rejeitado pelos estudiosos atuais de hipnose -, a maioria dos autores identificou-o com o fluido nervoso ou eletricidade animal, que muitos acreditavam estar por trás do funcionamento do sistema nervoso ${ }^{41,42}$.

Enquanto o magnetismo animal era visto por muitos como um princípio físico, havia representantes do mesmerismo que defendiam a existência de aspectos não físicos dos seres humanos. Eles sustentavam suas crenças por meio do relato de fenômenos exibidos por sujeitos mesmerizados, os quais incluíam situações como clarividência, conhecimento do pensamento de outros, diagnósticos médicos, entre outras manifestações.

Por exemplo, em seu livro Instruction pratique sur le magnétisme animal (1825), J. P. F. Deleuze (1753-1835)43, um bem conhecido defensor do magnetismo animal, disse que os fenômenos do sonambulismo magnético "oferecem uma prova direta da espiritualidade da alma" (p. 99). Ele definiu o sonambulismo como um estado semelhante ao sono e no qual a pessoa conseguiria conversar com o magnetizador. Mas, quando a pessoa retornava ao seu estado habitual, ela não reteria qualquer memória do que tinha ocorrido" (p. 98). A crença na natureza espiritual da condição (sonambulismo) foi baseada na interpretação que Deleuze fazia de suas observações. Ele via o sonambulismo como mostrando uma "distinção de duas substâncias, a dupla existência do interior do homem e do exterior do homem em um único indivíduo..." (p. 99).

Lawyer J. C. Colquhoun (1785-1854) argumentava, em Isis Revelata ${ }^{4}$, que o fenômeno do mesmerismo tal como a aquisição de conhecimento não previamente possuído pelo indivíduo mesmerizado proporcionaria evidências da existência da alma. Em sua 
visão, alguns dos fenômenos do magnetismo animal mostravam "uma prova impressionante da existência independente da alma do homem, e consequentemente, as mais sólidas base filosóficas para supor sua imortalidade..." (vol. 2, p. 166).

Muitos outros relacionavam o magnetismo animal à alma ${ }^{45,46}$. Vários defendiam que o magnetismo produzia um estado (sonambulismo magnético) que, por sua vez, liberava faculdades da alma usualmente obscurecidas na vida comum. Por exemplo, o magnetizador itinerante Charles Lafontaine (1803-1892) defendia que, quando o corpo se tornava inerte pela aplicação do magnetismo, a "vida do corpo é aniquilada, a alma... separada da vida comum para viver a sua própria vida. Suas faculdades, todas imateriais, mostram-se tão mais vívidas quanto maior é a inaquilação da matéria" 47 (p. 62). A visão sem o corpo, acreditava Lafontaine, era possível porque a alma estava separada do corpo.

Referindo-se aos fatos do mesmerismo, um autor comentava sobre a questão da sobrevivência à morte. De fato, ele parecia sugerir que os potenciais do mesmerismo permitiam-nos ver lampejos da vida espiritual futura, assumindo, por sua vez, um componente espiritual do ser humano durante a vida: "O homem está mostrando através desse de ser capaz de aumentar seu poder sensorial. Cui bono - com que finalidade, se após esse incremento de faculdade não se tornar permanente? Poderia ser isso consistente com a bondade da Providência para nos atormentar com vislumbres imperfeitos dos quais nós nunca seremos permitidos vivenciar? Poderiam as larvas terem rudimentos de asas se elas nunca fossem capazes um dia de voar? Nós não podemos pensar tão pobremente da sabedoria criativa ou de uma natureza parcimoniosa"48 (p. 533-534).

O autor e médico alemão Johann Heinrich Jung-Stilling (17401817) era da opinião que: "O magnetismo animal prova inegavelmente que nós temos um homem interno, uma alma, a qual é constituída de uma centelha divina, o espírito imortal, possuindo razão e vontade e um corpo luminoso, o qual é inseparável dele" 49 (p. 22). Tais ideias não eram científicas, mas foram baseadas em observações dos fenômenos, que os mesmeristas acreditavam ser inexplicáveis pelo conhecimento vigente.

\section{Espíritos e almas errantes}

Aparições de vivos eram frequentemente utilizadas para reforçar a ideia de que o espírito, a alma ou algum aspecto consciente do ser humano era capaz de funcionar fora do corpo ${ }^{50,51}$. Esses eram casos nos quais uma aparição, habitualmente uma representação visual de um indivíduo, era percebida por alguém quando a pessoa não estava fisicamente presente no local.

Enquanto alguns pesquisadores da SPR - tais como Edmund Gurney (1847-1888) e Frank Podmore (1856-1910) - defendiam que essas aparições representavam a exteriorização de mensagens adquiridas telepaticamente por um indivíduo que as capta ${ }^{52,53}$, outros assumiam a existência de um espírito capaz de viajar além do corpo carreando a consciência ${ }^{54,55}$. Essa pressuposição claramente assumia a independência de uma parte pensante dos seres humanos em relação ao funcionamento do corpo físico.

Posteriormente, muitos outros autores posteriores tiveram ideias semelhantes, mas com algumas variações. Esse foi o caso do engenheiro francês Gabriel Delanne (1857-1926) e do pesquisador italiano de fenômenos psíquicos Ernesto Bozzano (1862-1943). Eles publicaram muitos casos argumentando que esses sustentavam a ideia de que as aparições de vivos eram mais do que imaginação, pois elas eram verídicas ${ }^{54,55}$.

Baseado em casos cuidadosamente investigados por pesquisadores da SPR, Myers ${ }^{56}$ especulou que tipos diferentes de aparições de vivos, tais como aquelas que ocorriam espontaneamente enquanto uma pessoa estava morrendo ou planejando chegar a algum lugar, eram manifestações do espírito com diferentes graus de consciência. Ele acreditava que o lugar onde a pessoa era vista era uma modificação de uma "certa porção do espaço, não materialmente nem oticamente, mas de tal maneira que pessoas especialmente suscetíveis poderiam percebê-la" (vol. 1, p. xix-xx).
Em tempos mais recentes, experiências fora do corpo têm sido discutidas como fenômenos sugestivos de que a consciência pode funcionar fora do corpo físico ${ }^{57}$. Tal ideia tem sido apoiada com evidências de experiências verídicas, tais como aquelas na qual uma informação tem sido adquirida enquanto a pessoa estava fora do seu corpo, particularmente informações referentes a eventos distantes ${ }^{58}$. O tópico tem sido estudado em laboratório usando desenhos metodológicos nos quais a pessoa é solicitada a ir, durante uma experiência fora do corpo, a um lugar determinado para perceber uma informação colocada lá59. Enquanto apenas poucos estudos têm mostrado resultados positivos, esses achados indicam que essa é uma área promissora que merece pesquisas adicionais.

Outra linha de estudo relevante é a investigação das experiências de quase morte. Vários estudos têm mostrado que o fenômeno tem características estáveis ${ }^{60}$. Baseado em seu estudo desse fenômeno, o cardiologista holandês Pim Van Lommel61 tem especulado que a consciência está armazenada em um espaço não local como campos de ondas de informação" (p. 183). Nos Estados Unidos, o psiquiatra Bruce Greyson ${ }^{62}$ aborda as implicações dessa experiência quando afirma que elas desafiam o reducionismo materialista "perguntando como uma consciência tão complexa, incluindo atividade mental, percepção sensorial e memória podem ocorrer sob condições nas quais os modelos fisiológicos atuais da mente consideram isso impossível. Esse conflito entre um modelo materialista da identidade mente-cérebro e a ocorrência das experiências de quase morte sob condições de anestesia geral ou parada cardíaca é profundo e inevitável" (p. 43).

\section{Telepatia e clarividência como faculdades espirituais}

Em seu estudo sobre telepatia e outras experiências psíquicas o astrônomo Camille Flamarion (1842-1925)63 concluiu: "Esses fenômenos provam, eu penso, que a alma existe, e que ela esta dotada com faculdades até o presente momento desconhecidas... Um pensamento pode ser transmitido de uma mente para outra. Existem transmissões mentais, comunicações de pensamentos e fluxos psíquicos entre almas humanas" (p. 485).

Myers $^{56}$ via a telepatia como uma faculdade espiritual. Isso era uma indicação de uma dimensão espiritual transcendental que atuava em seres humanos subconscientemente e que representava uma faculdade que os seres humanos empregariam na outra dimensão.

Durante o século XX muitos outros viam a telepatia e a clarividência como evidência de um princípio não físico ${ }^{64,65}$. Um representante importante desse pensamento foi o bem conhecido parapsicólogo americano J. B. Rhine (1895-1980). Em seu livro The Reach of the Mind, Rhine ${ }^{66}$ não somente descreveu seu trabalho experimental com experiências extrassensoriais (e psicocinese), mas argumentou que os resultados dessa pesquisa mostravam que os fenômenos que ele estudava eram o resultado de um princípio não físico separado, mas interagindo com o corpo. Na sua visão a pesquisa de experiências extrassensoriais mostrava que a "mente pode escapar dos limites corporais sob certas condições... Nesse sentido, uma diferença distinta entre mente e matéria, um dualismo relativo, tem sido demonstrada..." (p. 205). Ele acreditava nisso porque nos seus experimentos as experiências extrassensoriais não tinham mostrado qualquer relação com variáveis físicas como distância e tempo, sendo esse último uma referência à precognição. De fato, Rhine ${ }^{67}$ referia-se à parapsicologia como uma ciência da natureza não física, uma disciplina envolvida com fenômenos "que falhavam em mostrar relações regulares com o tempo, espaço, massa e outros critérios fisicalistas" (p. 801). Em sua visão, a presença de tais componentes não físicos no homem validava aspectos da religião e poderia ser usada para combater o comunismo e o materialismo em geral ${ }^{68}$.

\section{Sobrevivência após a morte e mediunidade}

Muitos esforços passados (e alguns atuais) de pesquisadores em pesquisa psíquica têm sido direcionados para estudos empíricos sobre a sobrevivência após a morte. Diferente de especulações puramente 
religiosas e filosóficas, pesquisadores psíquicos têm obtido evidências empíricas para fenômenos tais como aparições e mediunidade (tópicos revisados por Braude ${ }^{69}$ ), que sugerem a alguns que a mente ou a consciência pode funcionar separada do corpo durante a vida e que pode continuar após a morte.

$\mathrm{Na}$ realidade, esse assunto de sobrevivência após a morte tem sido central para a pesquisa psíquica desde o começo dessa disciplina. Um dos principais fenômenos nesse sentido tem sido a mediunidade, ou a ideia de que certos indivíduos podem produzir mensagens provenientes dos mortos. A antiga literatura sobre pesquisa psíquica, tal como alguns estudos da médium americana Leonora E. Piper (1857-1950), apresenta um exemplo de estudos de sessões nas quais a sobrevivência do espírito tem sido considerada seriamente pelos pesquisadores psíquicos conhecidos por sua abordagem empírica rigorosa sobre o tópico ${ }^{70,71}$. Na mesma linha, houve muitos outros estudos relevantes com outros médiuns ${ }^{72,73}$.

Tem havido muitas outras linhas de pesquisa nas quais os fenômenos sugestivos de sobrevivência após a morte têm sido estudados empiricamente. Por exemplo, entre elas estão os casos de crianças que afirmam lembrar-se de vidas passadas e do estudo de aparições de mortos (revisados por Braude ${ }^{69}$ ).

\section{Considerações finais}

Neste artigo apresentei um breve panorama de discussões prévias de fenômenos psíquicos que apoiam a existência da mente, do espírito ou algum tipo de princípio não físico nos seres humanos. Minha revisão obviamente não cobriu todos os aspectos relevantes do tópico. Por exemplo, não abordei textos e pesquisas sobre visão a distância, casos de reencarnação, psicocinese, nem sobre efeitos da prece ou intenção terapêutica a distância. Além do mais, deve ser lembrado que dicotomias tais como material e imaterial, físico e não físico, são simples demais, particularmente quando levamos em consideração ideias da física moderna como discutido, por exemplo, em relação ao fenômeno psíquico $^{74}$.

As ideias apresentadas neste artigo são especulações para dar conta de fenômenos inexplicados. Como tais, elas não são aceitas por todos os estudiosos do assunto. Posto que essas sejam questões que não podem ser medidas diretamente, ficamos com interpretações sobre a não fisicalidade que carecem de detalhes relevantes sobre a natureza do processo, isto é, muitas das ideias discutidas sobre a natureza da mente ou do espírito são vagas, não indo além da afirmação de que os fenômenos não parecem ser explicáveis considerando meios físicos convencionais. Embora isso seja problemático e limite o progresso conceitual e da pesquisa na área, na realidade isso não é muito diferente de muitas especulações em ciência. Muitas áreas da ciência lidam com o estudo de fenômenos que não são compreendidos e então é necessário começar a partir de especulações baseadas no que é observável, um processo que também se aplica a ideias materialistas.

Mas independentemente dessas questões, o melhor dos esforços da pesquisa psíquica necessita ser reconhecido como um esforço sério e empírico para estudar propriedades da mente e como uma literatura que apresenta os fatos que não são facilmente explicados por meio de processos psicológicos, físicos e fisiológicos convencionais.

Embora acredite que exista evidência aceitável para sustentar a existência de fenômenos psíquicos, além de explicações convencionais como fraude, coincidência e alucinação, não foi a minha intenção neste artigo fornecer ou discutir essas evidências em detalhes. Em vez disso, apresentei um panorama de uma literatura e um conjunto de argumentos que merecem ser reconhecidos como parte da história intelectual e da história da filosofia e psicologia, campos relevantes ao problema mente-corpo. Infelizmente, muito do que mencionei é geralmente ignorado pela maioria daqueles que abordam esses tópicos e particularmente pelos que se dedicam a disciplinas relacionadas ao problema da mente-corpo. Tal fato é lamentável, posto que a omissão desse material dos relatos históricos prevalentes sobre o problema mente-corpo cria uma história incompleta do tema.
A mensagem discutida neste artigo tem sido expressa por outros recentemente a partir do ponto de vista da existência e validade dos fenômenos, os quais são concebidos como manifestações incapazes de ser explicadas pelo paradigma materialista ${ }^{75}$. Charles T. Tart tem nos lembrado sobre isso em seu livro The End of Materialism ${ }^{78}$, no qual ele postula que o fenômeno parapsicológico mostra que a abordagem fisicalista não consegue muitos aspectos importantes de nossa natureza ${ }^{76}$. Em suas palavras: "Os achados da parapsicologia científica forçam-nos a aceitar pragmaticamente que as mentes conseguem fazer coisas... que não podem ser reduzidas a explicações físicas, dado um conhecimento científico atual ou extensões razoáveis dele" (p. 241).

Esses autores, e muitos outros recentes, têm enfatizado a existência de uma mente não física, continuando a tradição intelectual descrita neste artigo. O psicólogo inglês John Beloff (1920-2006) ${ }^{77}$ caracterizou essa tradição como uma que "mostra a mente como um fator eficiente no mundo real, não somente como um epifenômeno sem valor e assim põe em questão a posição fisicalista” (p. 518).

\section{Referências}

1. Crane T, Patterson S, editors. History of the mind-body problem. London: Routledge; 2000.

2. MacDonald PS. History of the concept of mind: speculations about soul, mind and spirit from Homer to Hume. Aldershot, Hants, England: Ashgate; 2003.

3. McDougall W. Body and mind: a history and a defense of animism. New York: Macmillan; 1911.

4. Wright JP, Potter P, editors. Psyche and soma: physicians and metaphysicians on the mind-body problem from antiquity to Enlightenment. New York: Oxford University Press; 2000.

5. Dingwall EJ, editor. Abnormal hypnotic phenomena: a survey of nineteenth century cases. New York: Barnes and Noble; 1967-1968.

6. Podmore F. Modern spiritualism: a history and criticism. London: Methuen; 1902. (2 v.)

7. Goodrick-Clarke N. The Western esoteric traditions: a historical introduction. New York: Oxford University Press; 2008.

8. Watkins CS. History and the supernatural in medieval England. New York: Cambridge University Press; 2007.

9. Crowe C. The night-side of nature, or ghosts and ghost seers. London: T. Newby; 1848. (2 v.)

10. Kardec A. Le livre des esprits. Paris: E. Dentu; 1857.

11. Perty M. Die mystischen Erscheinungen der menschlichen Natur. Leipzig. C.F. Winter; 1861

12. Figuier L. Histoire du merveilleux dans des temps moderns. Paris: L. Hachette; 1860. (4 v.)

13. Inglis B. Natural and supernatural: a history of the paranormal from earliest times to 1914 (ed. rev.). Dorset: Prism; 1992.

14. Objects of the Society. Proc Soc Psych Res. 1882;1:36

15. Gauld A. The founders of psychical research. London: Routledge \& Kegan Paul; 1968.

16. Alvarado CS. Dissociation in Britain during the late nineteenth century: the Society for Psychical Research, 1882-1900. J Trauma Dissociation. 2002;3:9-33.

17. Brower MB. Unruly spirits: the science of psychic phenomena in modern France. Chicago: University of Illinois Press; 2010.

18. Wolffram $H$. The stepchildren of science: psychical research and parapsychology in Germany, c. 1870-1939. Amsterdam: Rodopi; 2009.

19. Myers FWH. Presidential address. Proc Soc Psychic Res. 1900;15:110-7.

20. James W. A record of observations of certain phenomena of trance (5). Part III. Proc Soc Psychic Res. 1890;6:651-9.

21. James W. Report on Mrs. Piper's Hodgson-control. Proc Soc Psychic Res. 1909;23:2-121.

22. Alvarado CS, Krippner S. Nineteenth century pioneers in the study of dissociation: William James and psychical research. J Conscious Stud. 2010;17:19-43.

23. Taylor E. William James on consciousness beyond the margin. Princeton: Princeton University Press; 1996.

24. James W. Human immortality. Boston: Houghton Mifflin; 1898. 
25. Krippner S, Friedman HL, editors. Debating psychic experience: human potential or human illusion? Santa Barbara, CA: Praeger; 2010.

26. Carpenter WB. Mesmerism and spiritualism, \&c. historically and scientifically considered. London: Longmans and Green; 1877.

27. Dendy WC. The philosophy of mystery. London: Longmans, Orme, Brown, Green \& Longmans; 1841.

28. Hall GS. Review of proceedings of the society for psychical research, and phantasms of the living. by E. Gurney, F.W.H. Myers, and F. Podmore. Am J Psychol. 1887;1:128-46.

29. Janet P. Lautomatisme psychologique: essai de psychologie expérimentale sur les formes inférieures de l'activité humaine. Paris: Félix Alcan; 1889.

30. Alvarado CS. Classic text $\mathrm{n}^{\circ}$ 84: 'Divisions of personality and spiritism' by Alfred Binet (1896). History Psychiatry. 2010;21:487-500.

31. Alvarado CS, Machado FR, Zangari W, Zingrone NL. Perspectivas históricas da influência da mediunidade na construção de ideias psicológicas e psiquiátricas. Rev Psiq Clín. 2007;34(Supp 1):42-53.

32. Le Maléfan P. Folie et spiritisme: histoire du discourse psychopathologique sur la pratique du spiritisme, ses abords et ses avatars (1850-1950). Paris: L'Hartmattan; 1999.

33. Moreira-Almeida A, Almeida AAS, Lotufo Neto F. History of 'spiritist madness' in Brazil. History Psychiatry. 2005;16:5-25.

34. Ellenberger HF. The discovery of the unconscious: the history and evolution of dynamic psychiatry. New York: Basic Books; 1970.

35. Binet A. Les altérations de la personnalité. Paris: Félix Alcan; 1892.

36. Crabtree A. From Mesmer to Freud: magnetic sleep and the roots of psychological healing. New Haven, CT: Yale University Press; 1993.

37. Plas R. Naissance d'une science humaine: la psychologie: les psychologues et le "merveilleux psychique". Rennes: Presses Universitaires de Rennes; 2000.

38. Gauld A. A history of hypnotism. Cambridge: Cambridge University Press; 1992.

39. Méheust B. Somnambulisme et mediumnité (1784-1930): Vol. 1: Le défi du magnétisme animal. Le Plessis-Robinson: Institut Synthélabo pour de Progrès de la Connaissance; 1999.

40. Mesmer F. Dissertation on the discovery of animal magnetism. In: Bloch G. editor. Mesmerism: a translation of the original scientific and medical writings of F. A. Mesmer. Los Altos, CA: William Kaufmann; 1980. (Original work published 1779). p. 43-78.

41. Charpignon J. Physiologie, médicine et métaphysique du magnétisme. Paris: Germer Baillière; 1848.

42. Esdaile J. Natural and mesmeric clairvoyance. London: Hippolyte Baillière; 1852.

43. Deleuze JPF. Instruction pratique sur le magnetisme animal. Paris: A. Belin; 1825 .

44. Colquhoun JC. Isis revelata: an inquiry into the origin, progress and present state of animal magnetism. 3rd ed. Edinburgh: Maclachlan \& Stewart; 1844. (2 v.)

45. Ashburner J. Notes and studies in the philosophy of animal magnetism and spiritualism. London: H. Baillière; 1867.

46. Haddock JW. Somnolism \& psycheism. 2nd ed. London: James S. Hodson; 1851.

47. Lafontaine C. L’art de magnétiser. 2nd ed. Paris: Germer Baillière; 1852.

48. Townsend $\mathrm{CH}$. Facts in mesmerism, with reasons for a dispassionate inquiry into it. London: Longman, Orme, Brown, Green \& Longmans; 1840.

49. Jung-Stilling JH. Theory of pneumatology, in reply to the question, what ought to be believed or disbelieved concerning presentiments, visions, and apparitions. New York: J. S. Redfield; 1834. (Original work published in 1808)

50. Alvarado CS. The spirit in out-of-body experiences: historical and conceptual notes. In: Batey B, editor. Spirituality, science and the pa- ranormal. Bloomfield, CT: Academy of Spirituality and Paranormal Studies; 2009. p. 3-19.

51. Owen RD. Footfalls on the boundary of another world: with narrative illustrations. Philadelphia: J.B. Lippincott; 1860.

52. Gurney E, Myers FWH, Podmore F. Phantasms of the living. London: Trübner; 1886. (2 v.)

53. Podmore F. Apparitions and thought-transference. London: Walter Scott; 1894.

54. Bozzano E. Les phénomènes de bilocation. Paris: Jean Meyer; 1937. (Original work published 1934)

55. Delanne G. Les apparitions matérialisées des vivants \& des morts: V. 1: Les fantômes de vivants. Paris: Librairie Spirite; 1909.

56. Myers FWH. Human personality and its survival of bodily death. London: Longmans, Green; 1903. (2 v.)

57. Woodhouse M. Out-of-body experiences and the mind-body problem. New Ideas Psych. 1994;12:1-17.

58. Hart H. ESP projection: spontaneous cases and the experimental method. J Amer Soc Psychical Res. 1954;48:121-46.

59. Alvarado CS. ESP during outofbody experiences: a review of experimental studies. J Parapsych. 1982;46:209-30.

60. Zingrone NL, Alvarado CS. Pleasurable Western adult near-death experiences: features, circumstances and incidence. In: Holden JM, Greyson B, James D, editors. The handbook of near-death experiences. Santa Barbara, CA: Praeger; 2009. p. 17-40.

61. Van Lommel P. Endless consciousness: a concept based on scientific studies on near-death experiences. In: Murray CD, editor. Psychological scientific perspectives on out-of-body and near-death experiences. New York: Nova Science; 2009. p. 171-86.

62. Greyson B. Implications of near-death experiences for a postmaterialist psychology. Psych Rel Spiritual. 2010;2:37-45.

63. Flammarion C. L'Inconnu: the unknown. New York: Harper \& Brothers; 1900.

64. Beloff J. The relentless question: Reflections on the paranormal. Jefferson, NC: McFarland; 1990.

65. Pratt JG. Parapsychology: an insider's view of ESP. New York: E.P. Dutton; 1967.

66. Rhine JB. The reach of the mind. New York: William Sloane; 1947.

67. Rhine JB. The science of nonphysical nature. J Phil. 1954;51:801-10.

68. Rhine JB. New world of the mind. New York: William Sloane; 1953.

69. Braude SA. Immortal remains: the evidence for life after death. Lanham, MD: Rowman \& Littlefield; 2003.

70. Hodgson R. A further record of observations of certain phenomena of trance. Proc Soc Psych Res. 1898;13:284-582.

71. Hyslop JH. A further record of observations of certain phenomena of trance. Proc Soc Psych Res. 1901;16:1-649.

72. Radclyffe-Hall M, Troubridge U. On a series of sittings with Mrs. Osborne Leonard. Proc Soc Psychic Res. 1919;30:339-554.

73. Beischel J, Schwartz GE. Anomalous information reception by research mediums demonstrated using a novel triple-blind protocol. Explore (NY). 2007;3:23-7.

74. Radin D. Entangled minds: extrasensory experiences in a quantum reality. New York: Paraview Pocket Books; 2006.

75. Kelly EF, Kelly EW, Crabtree A, Gauld A, Grosso M, Greyson B. Irreducible mind: toward a psychology for the 21st century. Lanham MD: Rowman \& Littlefield; 2007.

76. Tart CT. The end of materialism: How evidence of the paranormal is bringing science and spirit together. Oakland, CA: New Harbinger; 2009.

77. Beloff J. The mind-brain problem. J Scien Explor. 1994;8:509-22. 